\title{
Developing clinical practice guidelines for Chinese herbal treatment of polycystic ovary syndrome: A mixed-methods modified Delphi study
}

\author{
Lily Lai $^{*, 1}$, Andrew Flower ${ }^{1}$, Michael Moore ${ }^{1}$, George Lewith ${ }^{1}$
}

University of Southampton, Primary Care and Population Sciences, Aldermoor Health Centre, Aldermoor Close, Southampton, Hampshire SO16 5ST, United Kingdom Available online 14 March 2015

\section{KEYWORDS \\ Chinese herbal medicine; Polycystic ovary syndrome; Menstrual disorders; Delphi; Mixed methods}

\begin{abstract}
Summary
Objectives: Preliminary evidence suggests Chinese herbal medicine (CHM) could be a viable treatment option for polycystic ovary syndrome (PCOS). Prior to conducting a clinical trial it is important to consider the characteristics of good clinical practice. This study aims to use professional consensus to establish good clinical practice guidelines for the CHM treatment of PCOS.

Design and setting: CHM practitioners participated in a mixed-methods modified Delphi study involving three rounds of structured group communication. Round 1 involved qualitative interviews with practitioners to generate statements regarding good clinical practice. In round 2 , these statements were distributed online to the same practitioners to rate their agreement using a 7-point Likert scale, where group consensus was defined as a median rating of $\geq 5$. Statements reaching consensus were accepted for consideration onto the guideline whilst those not reaching consensus were re-distributed for consideration in round 3. Statements presented in the guidelines were graded from A (strong consensus) to D (no consensus) determined by median score and interquartile range.

Results: 11 CHM practitioners in the UK were recruited. After three Delphi rounds, 91 statement items in total had been considered, of which 89 (97.8\%) reached consensus and $2(2.2 \%)$ did not. The concluding set of guidelines consists of 85 items representing key features of $\mathrm{CHM}$ prescribing for PCOS.

Conclusions: These guidelines can be viewed as an initial framework that captures fundamental principles of good clinical practice for CHM.

(c) 2015 Elsevier Ltd. All rights reserved.
\end{abstract}

\footnotetext{
* Corresponding author. Tel.: +44 02380 241073; fax: +44 02390701125.

E-mail addresses: L.Y.W.Lai@southampton.ac.uk (L. Lai), flower.power@which.net (A. Flower), mvm198@southampton.ac.uk (M. Moore), GL3@southampton.ac.uk (G. Lewith).
}

1 Fax: +4402390 701125 . 


\section{Introduction}

Polycystic ovary syndrome (PCOS) affects $6-18 \%$ of women of reproductive age and is a heterogeneous condition characterised by endocrine and metabolic disturbances. ${ }^{1-3}$ Primary care management typically involves oral contraceptives and insulin-sensitising agents which have been associated with intolerable side-effects, poor adherence and increased metabolic risk. ${ }^{4-8}$ This highlights issues with current management and warrants exploration of other treatments that could be more acceptable.

Chinese herbal medicine (CHM) is used for symptoms of PCOS and there is emerging evidence from randomised controlled trials (RCTs) suggesting CHM could play an important role in the management of PCOS. ${ }^{9}$ However, many of these RCTs have been conducted in China and are methodologically poor thus limiting the generalisability of these findings. ${ }^{10}$ This necessitates further exploration of the effects of CHM with a fully-powered RCT in the UK. It is important however to ensure such a study is relevant to CHM practice in Western countries such as the UK, and that it takes into consideration characteristics of good clinical practice as determined by $\mathrm{CHM}$ practitioners. Accordingly, the aim of this study was to establish good clinical practice guidelines for the $\mathrm{CHM}$ treatment of PCOS.

\section{Materials and methods}

\section{Design}

We conducted a practitioner-consultation exercise using a modified Delphi method. The Delphi structures group communication to enable the views of informed individuals to be considered anonymously from each other and attempts to minimise the drawbacks of collective decision-making. It is particularly useful where diverse opinions exist and has other advantages as reducing the effect of prevailing opinions or dominant individuals, and enabling participants to alter previously expressed opinions without fear of losing face. ${ }^{11}$ It is a formal consensus method recognised by the UK's National Institute for Health and Clinical Excellence (NICE) for developing guidelines. ${ }^{12-14}$ It has also been successfully employed in a number of $\mathrm{CHM}$ and acupuncture studies for generating consensus in clinical practice or protocol development. ${ }^{15-19}$

Ethical approval was granted in June 2011 by the University of Southampton School of Medicine ethics committee (SOMSEC094.11).

\section{Recruitment}

We estimated 15 practitioners would be required to generate a sufficiently diverse range of views. ${ }^{16,18}$ Practitioners had to (1) have been in practice for a minimum of 4 years, (2) be a registered $\mathrm{CHM}$ practitioner in their respective country, (3) have self-reported expertise or interest in gynaecological conditions such as PCOS, (4) have access to the internet and to email, and (5) be fluent in English. To foster diversity in opinion, we purposively sampled for gender, country of CHM training, current country of practice and $\mathrm{CHM}$ practice style to include traditional Chinese medicine and classical styles of practice.

We used expert and snowball sampling methods by providing CHM professional bodies in the UK, US, Australia and Canada with a project brief, inviting suggestions of approximately 10 suitable practitioners. When practitioners were contacted, we asked for further suggestions of practitioners. A number of practitioners known to the research team were approached directly. Practitioners were provided an invitation letter, participant information sheet and completed a consent form prior to the study.

\section{Delphi rounds}

The Delphi involves iterative rounds of structured group communication. Round 1 consists of an 'idea-generating' exercise which typically involves participants independently producing a list of ideas or statements. In this study however, the lead author conducted in-depth qualitative interviews with each participant. This is a modification of the Delphi method which has been successfully carried out in previous studies and which in our view would maximise participant involvement. ${ }^{18,20}$

Interviews were conducted using a semi-structured interview guide which included open non-leading questions. Interviews were audio-recorded and transcribed verbatim by the lead author or an independent transcription service. All transcriptions were checked by the lead author. Additional field notes were taken and considered during analysis. Analysis was based on principles of thematic and framework analysis and conducted by coding in Microsoft Word 2010 using the 'Comments' function and charting in Microsoft Excel 2010. Framework analysis features a number of stages, involving familiarising self with the data, developing a thematic framework and charting and mapping to identify patterns within the data. ${ }^{21}$ This enabled a list of statements regarding clinical practice to be prepared for the next Delphi round.

In round 2, these statements were distributed back to the group using SurveyMonkey, a web-based questionnaire. Participants were asked to indicate their level of agreement with each statement using a 7-point Likert scale (' 1 strongly disagree' to ' 7 strongly agree'), including a score of ' 4 neither agree nor disagree'. We provided a response option of 'not applicable' (NA) for each item and a free-text comments box. Where participants had chosen to respond ' $N A$ ' to a particular statement, they were removed as a denominator in response to that item only. Demographic and clinical background information was also collected within this questionnaire.

Statistical analysis was conducted using Microsoft Excel 2010. Group consensus was defined a priori as a median group rating of 5 or more as used in previous Delphi studies. ${ }^{18}$ Following the Delphi method, items achieving consensus were accepted. Items not achieving consensus were circulated back to the participants for reconsideration in round 3. These were presented with the participant's original rating, the group rating and an invitation to re-consider their rating which they could accept or decline. Where a new rating was offered, this was used to reanalyse the group rating. Freetext comments from round 2 were analysed qualitatively and 


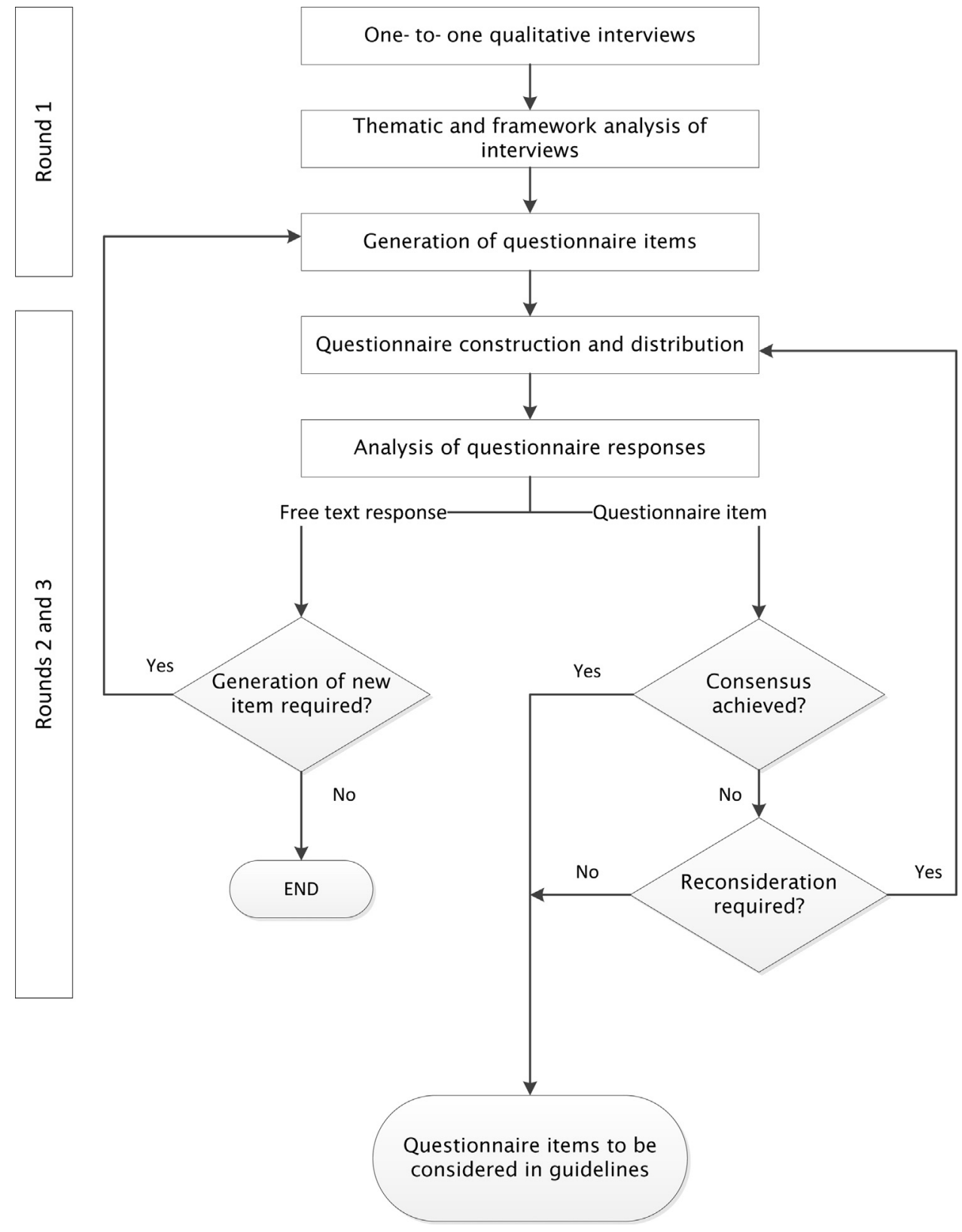

Figure 1 Overview of the Delphi process.

which could lead to the generation of new statements to be distributed in round 3 . We planned for each statement to be circulated a maximum of two times, and a maximum of four Delphi rounds to be conducted. An overview of the Delphi process can be viewed in Fig. 1.

\section{Clinical guidelines}

We regarded it important to present clinical practice points regardless of consensus status, and to illustrate consensus strength. We followed NICE guidance that grades strength of recommendation using the system Grading of Recommendations Assessment, Development and Evaluation (GRADE) [22]. The grading we adopted took into consideration the median ratings per item, and the interquartile ranges (IQRs) to reflect the levels of variability and therefore of opinion as demonstrated in earlier studies [14,23]. The criteria for determining grading were decided post hoc after statements had been distributed and are shown in Table 1.

Table 1 Criteria for grading clinical guideline items.

\begin{tabular}{llll}
\hline Grade & Description & Median & $\mathrm{IQR}^{\mathrm{a}}$ \\
\hline $\mathrm{A}$ & Strong consensus & 6 OR 7 & $\leq 2$ \\
$\mathrm{~B}$ & Moderate consensus & 6 OR 7 & $>2$ \\
& & 5 & $\leq 2$ \\
C & Weak consensus & 5 & $>2$ \\
D & Consensus not achieved & $<5$ & \\
\hline a $I$ IQR $=$ Interquartile range. & &
\end{tabular}


Table 2 Demographics and characteristics of recruited participants.

\begin{tabular}{ll}
\hline Characteristics & Number of participants (\%) \\
\hline Gender & $5(45.5)$ \\
Male & $6(54.5)$ \\
Female & $7(63.6)$ \\
CHM practice style & $1(9.1)$ \\
TCM only & $3(27.3)$ \\
Classical only & \\
Both TCM and Classical & $7(63.6)$ \\
Country of herbal training & $4(36.4)$ \\
UK & \\
China & $3(27.3)$ \\
Number of years in practice $($ mean $=18$, range $4-34$, \\
SD $=9.99)$ & $5(45.5)$ \\
$4-10$ & $1(9.1)$ \\
$11-20$ & $2(18.2)$ \\
$21-30$ & $0(0)$ \\
$31-40$ & $6(54.5)$ \\
Number of PCOS patients per month \\
0 & $4(36.4)$ \\
$1-4$ & $0(0)$ \\
$5-10$ & $1(9.1)$ \\
$11-20$ & \\
$>20$ & \\
\hline
\end{tabular}

\section{Results}

\section{Recruitment}

From 47 potentially eligible practitioners, 11 (23.4\%) were recruited. Fig. 2 shows the recruitment flowchart.

Demographics of the 11 participants are shown in Table 2. All participants were currently practicing in the UK as practitioners from Australia, US and Canada either did not respond, or declined to participate for reasons such as a lack of time and not feeling sufficiently experienced. Owing to lack of time and resources, we were unable to pursue recruitment in these countries. However, as shown in Table 2, we recruited a heterogeneous group of UK practitioners that was comparable to those from these countries in terms of training, CHM practice style, years in practice and experience [24-26].

\section{Delphi rounds}

For round 1, 10 face-to-face interviews and 1 telephone interview were conducted with a mean duration of 56 minutes (39-70 minutes). As these 11 interviews provided a sufficiently diverse range of opinions which was suitable for refinement within the Delphi process, no further interviews were required.

Qualitative analysis resulted in the generation of 85 statements covering 6 themes: Clinical presentation (11 items), Chinese medicine diagnosis (12 items), Chinese medicine treatment strategy ( 4 items), Chinese medicine interventions planning (16 items), Chinese herbal intervention planning (17 items) and Treatment assessment and evaluation (25 items). Fig. 3 shows how a statement was generated from interview data.

In round 2, a questionnaire containing these 85 statements were distributed back to the 11 participants, achieving a $100 \%$ response rate within 8 weeks. Group consensus was achieved in 83 items (97.6\%) whilst 2 items $(2.4 \%)$ failed to reach consensus. Comments were made in 13 of the 16 free text boxes by at least one participant and were added to the initial data corpus for further analysis. 'NA' was a response for 1 item and for one participant only and who was subsequently removed as a denominator in the analysis of this item.

In round 3, a questionnaire was distributed containing 8 items; 2 items that had failed to reach consensus, 4 new items generated from free-text responses and 2 clarified items. The 2 clarified items originated from 1 item that reached group consensus in round 2 but which had received comments regarding lack of clarity. We decided this should be separated into 2 new items for consideration. This questionnaire achieved $100 \%$ response within 3 weeks. For one of the two re-distributed items, 5 participants chose to re-rate the item. Analysis taking into consideration the new ratings indicated group consensus was not achieved. For the second re-distributed item, all 11 participants declined the offer to change their ratings. No further redistribution of these two items was therefore required. For the four new items and two clarified items, group consensus was achieved.

In summary after three Delphi rounds comprising one interview round and two questionnaire rounds over 7 months, a total number of 91 unique statement items had been distributed for consideration by the panel, of which 89 $(97.8 \%)$ reached consensus and 2 (2.2\%) did not.

\section{Guideline presentation}

From the 91 unique statement items, 83 were presented in the guideline. To improve clarity, two items were combined to form one item in the guideline. One item was discarded as it had been replaced with two new items. Six items were discarded to avoid repetition.

The concluding set of guidelines consists of 85 items: 83 guideline items presented with a grade indicating strength of group consensus and two clinical practice items reporting dosage ranges that were not graded as consensus had not been sought. Fig. 4 shows a section of the guidelines 'Chinese Herbal Intervention Planning'. The complete guideline can be seen in Appendix 1.

\section{Discussion}

This study adopted a modified Delphi approach to facilitate a systematic and rigorous practitioner-consultation exercise with 11 UK CHM practitioners, resulting in the development of good clinical practice guidelines. These guidelines consist of 85 items illustrating key features of CHM-prescribing for PCOS, 83 of which have been assigned a consensus grade. These describe PCOS symptoms and signs seen in clinical practice, common CM diagnoses and practice points regarding how treatment is managed and evaluated by $\mathrm{CHM}$ practitioners. These clinical perspectives can now be compared with other published work and used to inform future 


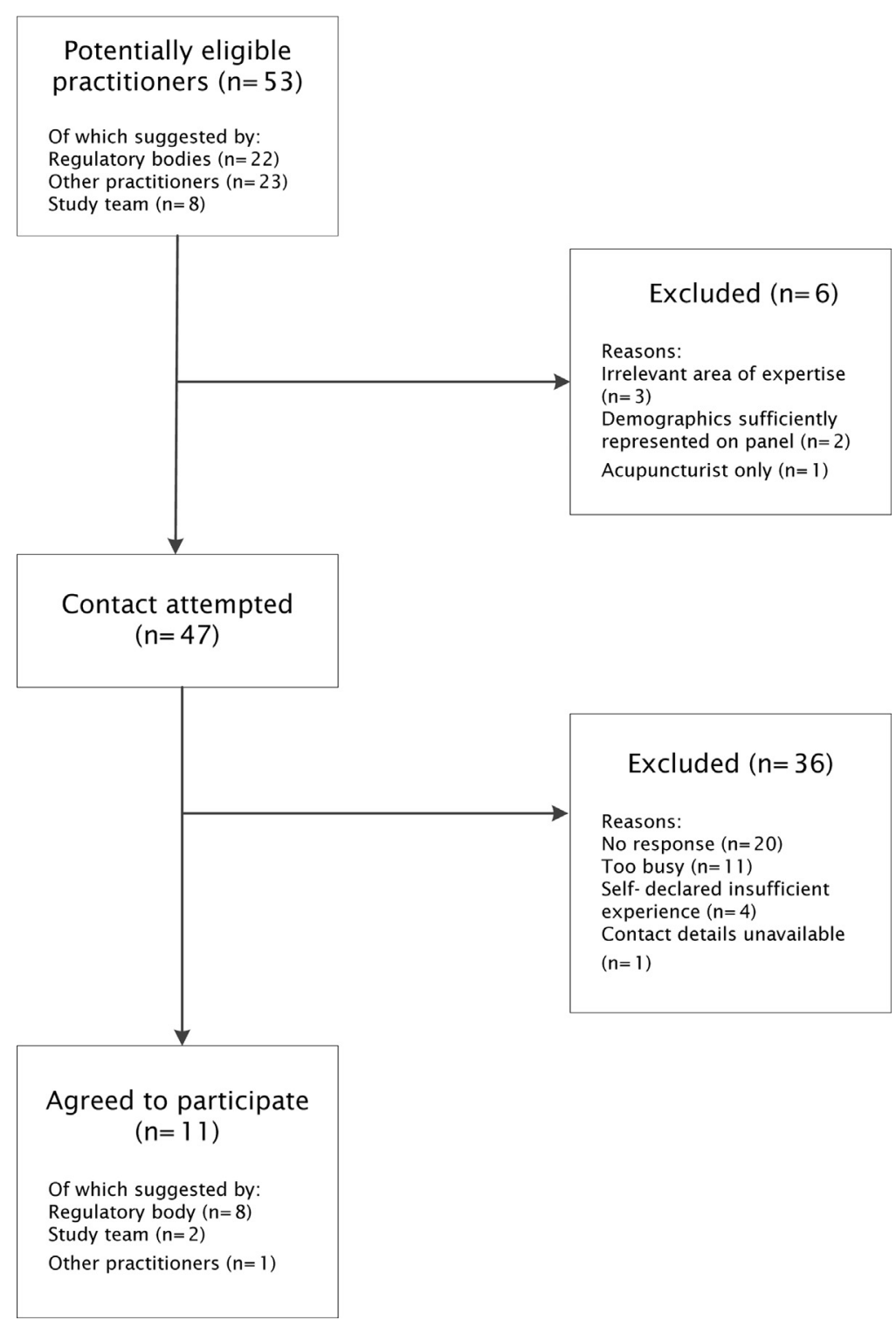

Figure 2 Flowchart of recruitment process.

studies. For example the RCT interventions described in a Cochrane review for PCOS-related subfertility focused on diagnostic patterns of Kidney yang deficiency and Spleen Qi deficiency. ${ }^{10}$ Although these were reported in our guidelines, other patterns were also identified such as Excess Cold in the Uterus and Blood deficiency that were not reported in the RCTs. This may be because these patterns occur more frequently in oligo- and amenorrhoea than in subfertility or because these patterns do not occur sufficiently frequently in the PCOS population to warrant investigation in RCTs. We hope this demonstrates how clinical knowledge drawn from the Delphi exercise can be triangulated and compared with current evidence as a way of maximising model validity.

A strength of this study is the use of a mixed-methods approach. The content of our guidelines had been generated through qualitative interviews, instead of basing items solely on published research or the research team's experience. This ensured guideline items were generated inductively and grounded in real-world practice by $\mathrm{CHM}$ practitioners independent from the research team. A further strength was our $100 \%$ response rate. Dropout between rounds is a widely acknowledged criticism of Delphi studies and although qualitative interviews have been used in previous studies, ${ }^{18,27}$ we feel our decision to conduct in-depth interviews with all participants contributed greatly to participant retention and which strengthens the credibility of this study. Finally, we recruited a heterogenous group of practitioners with criteria determined a priori. This enabled us to consider the views of a diverse group of practitioners and thus maximise the relevance of our findings across CHM practice in the West. Of particular note is the diversity of CHM practice styles within our panel to represent the range of styles found in other countries, rather than focusing only on the dominant TCM style.

Owing to language restrictions and a lack of resources, we required practitioners to be fluent in English which means the views of those fluent only in Chinese may not have been adequately represented. However, we included the purposive sampling criterion 'country of CHM training' 


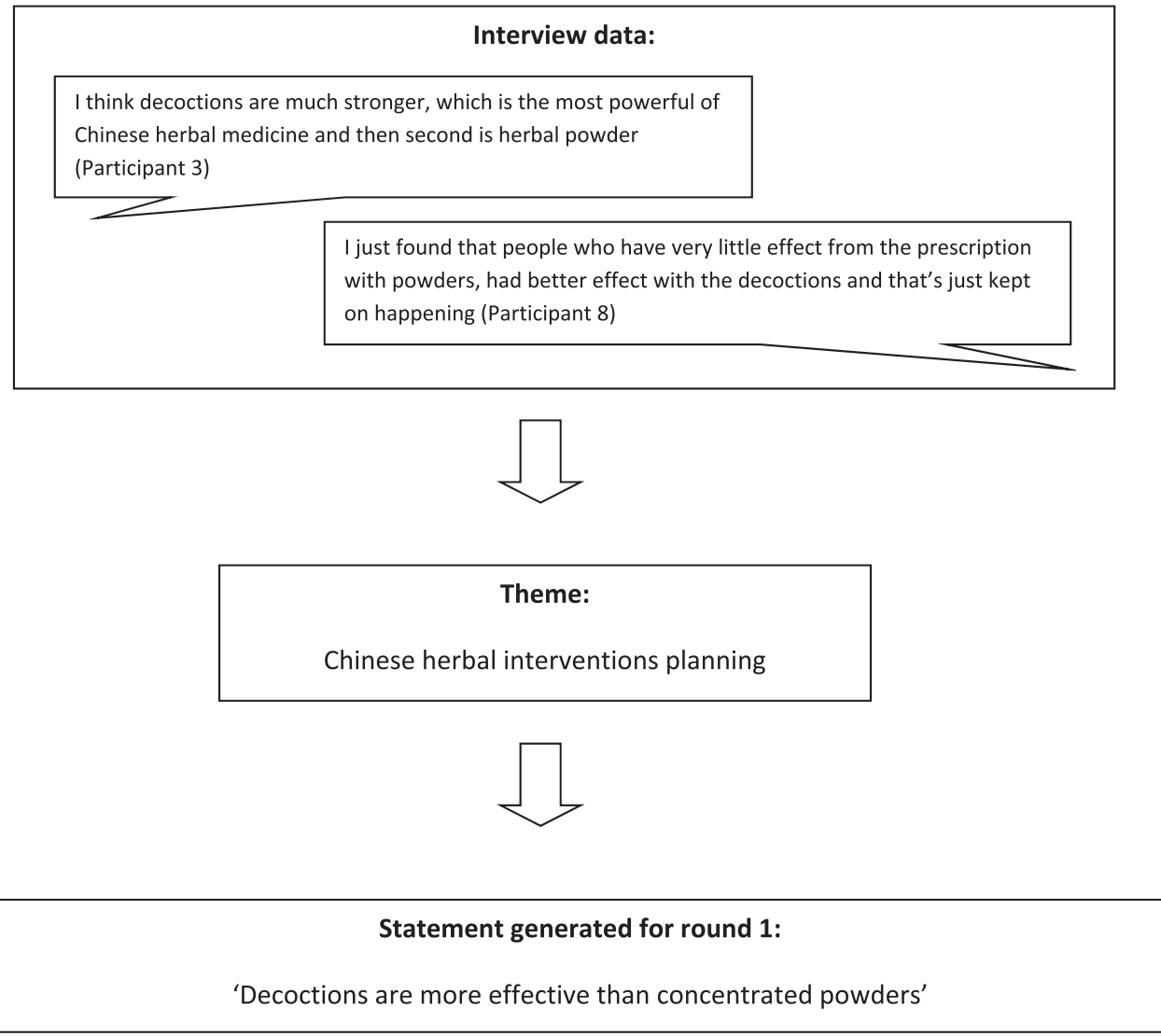

Figure 3 Generation of a statement from interview data.

\section{Chinese Herbal Intervention Planning}

Consensus

Grade

5.1. Decoctions are more effective than concentrated powders

A

5.2. Concentrated powders are suitably effective for PCOS

A

5.3. Individualised herbs are more effective than standardised herbs

A

5.4. Individualisation is important:

5.4.1. To adapt to the specific needs of the patient

A

5.4.2. To prevent resistance to a herbal prescription

C

5.5. In Blood stasis, herbs for removing Blood stasis should be prescribed for

use during menses

5.6. Whilst patients are actively trying to conceive:

5.6.1. Blood stasis removing herbs should not be prescribed after ovulation

5.6.2. It is suitable to prescribe Blood stasis removing herbs after ovulation, IF patients also present with a Blood stasis condition

5.7. The prescribed daily dosage range of concentrated powders is $5 \mathrm{~g}$ to $25 \mathrm{~g}$

5.8. The most frequently prescribed daily dosage range of concentrated

powders is $5 \mathrm{~g}$ to $15 \mathrm{~g}$.

PCOS=Polycystic ovary syndrome; Consensus grade: $A$ = Strong consensus; $B=$ Good consensus; $C$ = Weak consensus; $D=$ No consensus, $N A=$ Not Applicable, formal consensus not sought

Figure 4 Excerpt 'Chinese Herbal Intervention Planning' from concluding clinical guidelines. 
and successfully recruited four bilingual practitioners who had trained and practiced in China. We deemed this to be sufficient representation for the purpose of this study. We were also unsuccessful in recruiting from a range of Western countries as intended due to limited time and resources. Despite this, we successfully recruited a heterogenous group of UK practitioners whose characteristics were comparable to those in other Western countries and thus we feel are representative of Western CHM practice. ${ }^{24-26}$ Researchers wishing to recruit overseas practitioners in future studies could benefit from allowing more time and flexibility during the recruitment process. We would also advise researchers to cost-in modest reimbursement for practitioner-time as an additional way of maximising recruitment.

To our knowledge, this is the first time the Delphi method has been successfully used to present good clinical practice guidelines relating to real-world CHM-prescribing for PCOS. Unique to this Delphi study is the presentation of consensus grading within our guidelines which provides a clear and accessible set of statements. We chose to adopt this in the spirit of NICE guideline development standards which assess the strength of recommendations using GRADE and seek to convey information in a transparent manner. ${ }^{22}$ Our aim was to use a similarly simple grading system to maximise the relevance and applicability of our guidelines to clinical practice and research. By ensuring that research evidence is based on real-world clinical practice, these guidelines form the first step towards maximising model validity in $\mathrm{CHM}$ research. This also promotes transparency as it enables researchers to justify decisions regarding study design and readers to critique research based on clinical perspectives that have been drawn out prior to a study commencing. These guidelines should not be seen as a definitive checklist but rather as an initial mapping exercise of key practice points in $\mathrm{CHM}$ prescribing. In the absence of population-level data from clinical and $\mathrm{CHM}$ prescription records and the paucity of $\mathrm{CHM}$ studies conducted in Western populations, these guidelines remain an important step towards documenting an initial framework of best practice.

\section{Conclusions}

We have successfully used a modified Delphi approach to facilitate a systematic and rigorous practitionerconsultation exercise, resulting in the development of good clinical practice guidelines for CHM treatment of PCOS. These guidelines were used in a subsequent clinical trial as the basis for good practice within the study protocol ${ }^{28}$ and can be viewed as an initial framework for good clinical practice to be updated as necessary. They provide a sufficiently broad scope to capture fundamental principles of good clinical practice whilst being appropriately detailed to be useful for practitioners and researchers alike.

\section{Financial support}

Yes.

\section{Conflict of interest}

None.

\section{Acknowledgements}

This study is supported by a PhD research training fellowship awarded to the main author by the National Institute for Health Research (NIHR) School for Primary Care Research (SPCR) (RCS Ref 10-0894). The NIHR SPCR is a partnership between the Universities of Birmingham, Bristol, Keele, Manchester, Nottingham, Oxford, Southampton and University College London. The views expressed are those of the authors and not necessarily those of the NHS, the NIHR or the Department of Health.

We are grateful to the practitioners who generously donated their time and insight during this research study: Laurie Ayres, Naava Carman, Jennie Dallas, Zhihua Gao, Jill Glover, Dan Jiang, Martin Logue, Alex Owen, Trevor Wing, Jidong Wu and Liqin Zhao. Other than the role stated, those named have had no other contribution to the study design, analysis or writing of the protocol and of this report.

\section{Appendix 1. Good clinical practice guidelines for the treatment of polycystic ovary syndrome with Chinese herbal medicine}

This clinical guideline has been developed by researchers at the University of Southampton using a practitionerconsultation exercise following the Delphi method. This guideline describes key features of good clinical practice that were developed with a panel of 11 herbalists practicing in the UK on the topic of Chinese herbal medicine (CHM) prescribing for polycystic ovary syndrome (PCOS).

For clinical practice items where consensus has been sought, these have been graded as follows:

$A=$ Strong consensus; $B=$ Good consensus; $C=$ Weak consensus; $D=$ No consensus

For clinical practice items where formal consensus was not sought and which describe a range of opinions, these have been summarised and graded 'NA'.

Chinese medicine (CM) here includes acupuncture, Chinese herbal medicine, moxibustion and tuina.

Clinical practice items

Consensus

1. Clinical presentation

1.1 In the clinic, patients with PCOS present

with one or more of the following signs and symptoms:

1.1.1. Delayed periods (cycles longer than 35 days)

1.1.2. Absent periods (no period for 6

months or more)

1.1.3. Infertility

1.1.4. Acne

1.1.5. Hirsutism (excessive facial and body hair)

1.1.6. Pre-menstrual syndrome (PMS) A

1.1.7. Emotional issues

1.1.8. Weight management issues

1.1.9. Heavy periods

1.1.10. Painful periods

1.2. PCOS is diagnosed as a result of fertility investigations
A

A

A

A

A

A

A

B

C

C 
2. Chinese medicine diagnosis

2.1. Treatments in Chinese medicine are based on pattern differentiation

2.2. In PCOS, combined excess and deficiency patterns are found

2.3. In PCOS, knowledge of a patient's biomedical status, such as status of ovaries or testosterone levels, informs the Chinese medicine treatment

2.4. The following excess patterns are found in PCOS:

2.4.1. Phlegm Damp accumulation

2.4.2. Blood stasis

2.4.3. Liver Qi stagnation

2.4.4. Excess Cold in the Uterus

2.4.5. Stagnated Liver Heat

2.5. The following deficiency patterns are found in PCOS:

2.5.1. Kidney Yang deficiency

2.5.2. Spleen Qi deficiency

2.5.3. Spleen Yang deficiency

2.5.4. Blood deficiency

2.5.5. Kidney Yin deficiency

3. Chinese medicine treatment strategy

3.1. Chinese medicine is an integrated system of interventions and self-care measures tailored to the individual

3.2. With combined excess and deficiency patterns in PCOS, Chinese medicine treatments should address both simultaneously

3.3. In PCOS, when excess patterns start to clear, treatments should prioritise nourishing deficiencies

3.4. In PCOS, treatments should initially prioritise clearing excess patterns

4. Chinese medicine interventions planning

4.1 When providing Chinese medicine treatment for PCOS, one or more of the following are recommended:

4.1.1. Chinese herbs

4.1.2. Acupuncture

4.1.3. Stress management techniques

4.1.4. Dietary changes

4.1.5. Appropriate work-life balance

4.1.6. Advice on exercise

4.2 Chinese herbs have the following

advantages over acupuncture:

4.2.1 More effective for chronic conditions 4.2.2 More effective for severe patterns of deficiency

4.2.3 Have a longer-lasting effect

4.2.4 More effective for hormonal conditions 4.2.5. More effective for severe patterns of excess

4.2.6. Can be administered more readily on a daily basis

4.3. Acupuncture has the following advantages over Chinese herbs:

4.3.1. Provides rapid relief of pain
4.3.2. Moves Qi rapidly

4.3.3. More effective for emotional issues

4.3.4. Provides direction for $\mathrm{Qi}$
A

A

A

A

A

A

A

A

A

A

B

C

A

A

A

D

A

A

A

A

A

A

A

A

A

A

A

A

A acupuncture is effective

4.5. In PCOS, both acupuncture and CHM are necessary to provide effective treatment

4.6. In PCOS, acupuncture only i.e. without $\mathrm{CHM}$ is effective

5 Chinese herbal intervention planning

5.1. Decoctions are more effective than concentrated powders

5.2. Concentrated powders are suitably effective for PCOS

5.3. Individualised herbs are more effective than standardised herbs

5.4. Individualisation is important:

5.4.1. To adapt to the specific needs of the patient

5.4.2. To prevent resistance to a herbal prescription stasis should be prescribed for use during menses

5.6. Whilst patients are actively trying to conceive:

5.6.1 Blood stasis removing herbs should not be prescribed after ovulation

5.6.2 It is suitable to prescribe Blood stasis also present with a Blood stasis condition

5.7. The prescribed daily dosage range of concentrated powders is $5 \mathrm{~g}$ to $25 \mathrm{~g}$

5.8. The most frequently prescribed daily to $15 \mathrm{~g}$.

6 Treatment assessment and evaluation

6.1. Chinese herbs are effective for the following PCOS-related symptoms:

6.1.1. Delayed periods (cycles longer than 35 days)

6.1.2. Infertility

6.1.3. Absent periods (no period for 6 months or more)

6.1.4. Heavy periods

6.1.5. Pre-menstrual syndrome (PMS)

6.1.6. Acne hair)

6.1.8. Painful periods

6.1.9. Emotional issues

6.1.10. Weight management issues is determined by assessing one or more of the following:

6.2.1. Cycle lengths

6.2.2. Heaviness of menstrual flow

6.2.3. Duration of menstrual bleeding

6.2.4. Menstrual clotting

6.2.5. Period pain
A

B

B

A

5.5. In Blood stasis, herbs for removing Blood removing herbs after ovulation, IF patients dosage range of concentrated powders is $5 \mathrm{~g}$

6.1.7. Hirsutism (excessive facial and body

6.2. In PCOS, initial response to Chinese herbs
A

D

A

A

A

A

A

A

A

A

A

B

A

A

A

A

A 
6.2.6. Colour of menstrual blood

6.2.7. Pulse palpation

6.2.8. Basal body temperature chart

6.2.9. Emotional wellbeing

6.2.10. Abdominal palpation

6.2.11. Tongue appearance

6.3. Response to Chinese herbs is slower in:

6.3.1. Phlegm Damp accumulation conditions

6.3.2. Overweight patients

6.3.3. Blood stasis conditions

6.4. When regulating menstruation in PCOS, a regular menstrual cycle is the observation of three consecutive menstrual cycles, each 35 days or less in length

6.5. In PCOS, an initial response to Chinese herbs is observed within 3 months

6.6 When prescribing Chinese herbs for delayed periods in PCOS (cycles longer than 35 days), a regular menstrual cycle is observed within 6 months

6.7. When prescribing Chinese herbs for absent periods in PCOS (no period for 6 months or more), a regular menstrual cycle is observed within 9 months

\section{References}

1. Asuncion M, Calvo RM, San Millan JL, Sancho J, Avila S, EscobarMorreale. HF. A prospective study of the prevalence of the polycystic ovary syndrome in unselected Caucasian women from Spain. J Clin Endocrinol Metab 2000;85(7):2434-8.

2. March WA, Moore VM, Willson KJ, Phillips DI, Norman RJ, Davies MJ. The prevalence of polycystic ovary syndrome in a community sample assessed under contrasting diagnostic criteria. Hum Reprod 2010;25(2):544-51.

3. Ehrmann DA. Polycystic ovary syndrome. N Engl J Med 2005; 352(12):1223-36.

4. Sills ES, Perloe M, Tucker MJ, Kaplan CR, Genton MG, Schattman GL. Diagnostic and treatment characteristics of polycystic ovary syndrome: descriptive measurements of patient perception and awareness from 657 confidential self-reports. BMC Womens Health 2001;1(1):3.

5. Fleming R, Hopkinson ZE, Wallace AM, Greer IA, Sattar N. Ovarian function and metabolic factors in women with oligomenorrhea treated with metformin in a randomized double blind placebo-controlled trial. J Clin Endocrinol Metab 2002;87(2): 569-74.

6. Donnan PT, MacDonald TM, Morris AD. Adherence to prescribed oral hypoglycaemic medication in a population of patients with Type 2 diabetes: a retrospective cohort study. Diabet Med 2002;19(4):279-84.

7. Grant RW, Devita NG, Singer DE, Meigs JB. Polypharmacy and medication adherence in patients with type 2 diabetes. Diabetes Care 2003;26(5):1408-12.

8. Legro RS, et al. Clomiphene, metformin, or both for infertility in the polycystic ovary syndrome. N Engl J Med 2007;356(6): 551-66.

9. Raja-Khan N, Stener-Victorin E, Wu X, Legro RS. The physiological basis of complementary and alternative medicines for polycystic ovary syndrome. Am J Physiol Endocrinol Metab 2011;301(1):E1-10.

10. Zhang J, et al. Chinese herbal medicine for subfertile women with polycystic ovarian syndrome. Cochrane Database Syst Rev 2010;9:CD007535.

11. von der Gracht HA. Consensus measurement in Delphi studies: review and implications for future quality assurance. Technol Forecast Soc Change 2012;79(8):1525-36.

12. Jones J, Hunter D. Consensus methods for medical and health services research. Br Med J 1995;311(7001):376-80.

13. National Institute for Health and Clinical Excellence. The guidelines manual; 2009.

14. Linstone HA, Turoff M. In: Linstone HA, Turoff M, editors. The Delphi method: techniques and applications. 2002.

15. Smith CA, Grant S, Lyttleton J, Cochrane S. Using a Delphi consensus process to develop an acupuncture treatment protocol by consensus for women undergoing Assisted Reproductive Technology (ART) treatment. BMC Complement Altern Med 2012;12:88.

16. Alraek T, Borud E, White A. Selecting acupuncture treatment for hot flashes: a Delphi consensus compared with a clinical trial. J Altern Complement Med 2011;17(1):33-8.

17. Zhao J, Zha Q, Jiang M, Cao H, Lu A. Expert consensus on the treatment of rheumatoid arthritis with Chinese patent medicines. J Altern Complement Med 2013;19(2):111-8.

18. Flower A, Lewith GT, Little P. Seeking an oracle: using the Delphi process to develop practice guidelines for the treatment of endometriosis with Chinese herbal medicine. J Altern Complement Med 2007;13(9):969-76.

19. Smith CA, Betts D. The practice of acupuncture and moxibustion to promote cephalic version for women with a breech presentation: implications for clinical practice and research. Complement Ther Med 2014;22(1):75-80.

20. Hasson F, Keeney S, McKenna. H. Research guidelines for the Delphi survey technique. J Adv Nurs 2000;32(4):1008-15.

21. Ritchie J, Spencer L. Qualitative data analysis for applied policy research. In: Bryman AB, Burgess RG, editors. Analyzing qualitative data. Routledge: Abingdon; 1994. p. 173-94.

22. Guyatt GH, et al. GRADE: an emerging consensus on rating quality of evidence and strength of recommendations. $\mathrm{Br}$ Med $\mathrm{J}$ 2008;336(7650):924-6.

23. Tan J, et al. Acne severity grading: determining essential clinical components and features using a Delphi consensus. J Am Acad Dermatol 2012;67(2):187-93.

24. Wang ZG. Job analysis report. National Certification Commission for Acupuncture and Oriental Medicine; 2013.

25. Allied Health Workforce. A.I.o.H.a. Welfare. Editor 2012:Camberra.

26. Bensoussan A, Myers SP. Towards a safer choice: The practice of traditional Chinese medicine in Australia. Campbelltown NSW: Faculty of Health, University of Western Sydney Macarthur; 1996.

27. Cochrane S, Smith CA, Possamai-Inesedy. A. Development of a fertility acupuncture protocol: defining an acupuncture treatment protocol to support and treat women experiencing conception delays. J Altern Complement Med 2011;17(4): 329-37.

28. Lai L, Flower A, Moore M, Prescott P, Lewith G. Polycystic ovary syndrome: a randomised feasibility and pilot study using Chinese Herbal medicine to explore Impact on Dysfunction (ORCHID) - Study protocol. European Journal of Integrative Medicine 2014. 